\title{
Dose Escalation Assessment Among Targeted Immunomodulators in the Management of Inflammatory Bowel Disease
}

\author{
Rahel Ehrenberg, BS; Jenny Griffith, PharmD; Cindy Theigs, RPh; and Bryan McDonald, BS
}

\begin{abstract}
BACKGROUND: The need for individualized treatment regimens is becoming more important in the management of patients with inflammatory bowel disease (IBD). Gastroenterologists may dose adjust either by increasing the dose or shortening the dosing interval from the initial recommended maintenance dose to achieve an appropriate clinical response. Understanding the role of dose escalation in the treatment of IBD in clinical practice provides payers in the United States insight into the real-world cost-effectiveness of targeted immunomodulators (TIMs) in the management of IBD.
\end{abstract}

OBJECTIVE: To assess the prevalence and magnitude of dose escalation for approved IBD therapies.

METHODS: Using the Source Healthcare Analytics database, patients with IBD who initiated treatment with a drug of interest from July 2015 to June 2017 were identified. Patient utilization of the TIMs was tracked for 12 months following initiation. All included patients had at least 2 diagnoses for ulcerative colitis or Crohn disease before TIM initiation and at least 5 claims for a drug of interest within the 12 months following initiation. Dose escalation was defined as an increase of at least $30 \%$ in the average daily dose (ADD) relative to the patient's expected maintenance dose on 2 consecutive prescriptions. The proportion of patients with dose escalation in the first 12 months after treatment initiation was determined. The magnitude of dose escalation was determined by calculating the patient's ADD across all noninduction dose claims and comparing it with the expected daily dose. Dose escalation prevalence and magnitude were used to quantify the equivalent patient treatment rate representing the number of patients per 100 that could have been treated with standard dosing, given the prevalence of dose escalation in the treated population.

RESULTS: 7,028 patients (2,406 infliximab, 1,966 adalimumab, 1,745 vedolizumab, 472 ustekinumab, 285 certolizumab pegol, and 154 golimumab) met eligibility criteria and were included in the study. Among IBD therapies, dose escalation occurred most frequently with infliximab (39\%), followed by adalimumab (28\%), vedolizumab (23\%), ustekinumab (22\%), certolizumab pegol $(20 \%)$, and golimumab (14\%). The magnitude of dose escalation was greatest for ustekinumab (131\%), followed by infliximab (70\%), vedolizumab $(62 \%)$, adalimumab (59\%), certolizumab pegol (50\%), and golimumab (45\%). The calculated patient equivalence was highest for infliximab (128) and ustekinumab (128) compared with adalimumab (116), vedolizumab (114), certolizumab pegol (110), and golimumab (106).

CONCLUSIONS: Among patients with IBD, dose escalation occurred with all TIMs examined with varying degrees of prevalence and magnitude. Realworld utilization patterns of TIMs indicate that dose escalation is an important part of the clinical management of IBD and needs to be considered when evaluating the cost-effectiveness of IBD treatments.

J Manag Care Spec Pharm. 2020;26(6):758-65

Copyright $\odot 2020$, Academy of Managed Care Pharmacy. All rights reserved.

\section{What is already known about this subject}

In patients with inflammatory bowel disease (IBD), dose escalation of therapy may occur to aid patients in achieving and/or maintaining disease remission.

Dose escalation is a key issue for payers as increasing the dose or dosing at shortened intervals frequently results in increased costs.

\section{What this study adds}

Dose escalation in IBD occurred with all targeted immunomodulators evaluated in this study with varying degrees of prevalence and magnitude.

On average, infliximab- and ustekinumab-treated patients with IBD consumed more medication than patients with IBD on other therapies based on the prevalence and magnitude of dose escalation.

Ј nflammatory bowel diseases (IBDs), including ulcerative colitis (UC) and Crohn disease (CD), are chronic, relapsing-remitting inflammatory conditions involving the gastrointestinal tract with possible genetic, immunologic, and environmental influences. ${ }^{1,2}$ IBD is progressive in nature; longterm complications include fistulas, abscesses, strictures, perforation of the bowel, fulminant colitis, toxic megacolon, and colorectal cancer, ${ }^{2-9}$ and all of these complications can lead to loss of intestinal function. The chronic and relapsing nature of IBD has a substantial negative effect on health-related quality of life and work productivity and is associated with high rates of fatigue, depression, and anxiety, especially during relapse..$^{10-18}$ The goal of the treatment is the induction and maintenance of remission to avoid complications., ${ }^{1,2}$

Targeted immunomodulators (TIMs) are used to treat patients with moderate to severe disease or those whose disease is refractory to other treatments. ${ }^{1,2}$ These agents include antitumor necrosis factor monoclonal antibodies (anti TNFs), interleukin 12/23 inhibitors, integrin receptor antagonists, and JAK inhibitors. ${ }^{19,20}$ Despite the availability of TIMs, not all patients with CD or UC are able to achieve or maintain remission. Approximately $10 \%$ to $30 \%$ of patients with IBD fail to respond to induction therapy with anti-TNF agents. ${ }^{21} \mathrm{~A}$ recent meta-analysis examined loss of response to anti-TNF maintenance therapy among patients with CD and found that with a median follow-up of 1 year, 30\%-41\% of patients experienced a loss of response, ${ }^{22}$ and the annual risk for loss of response 


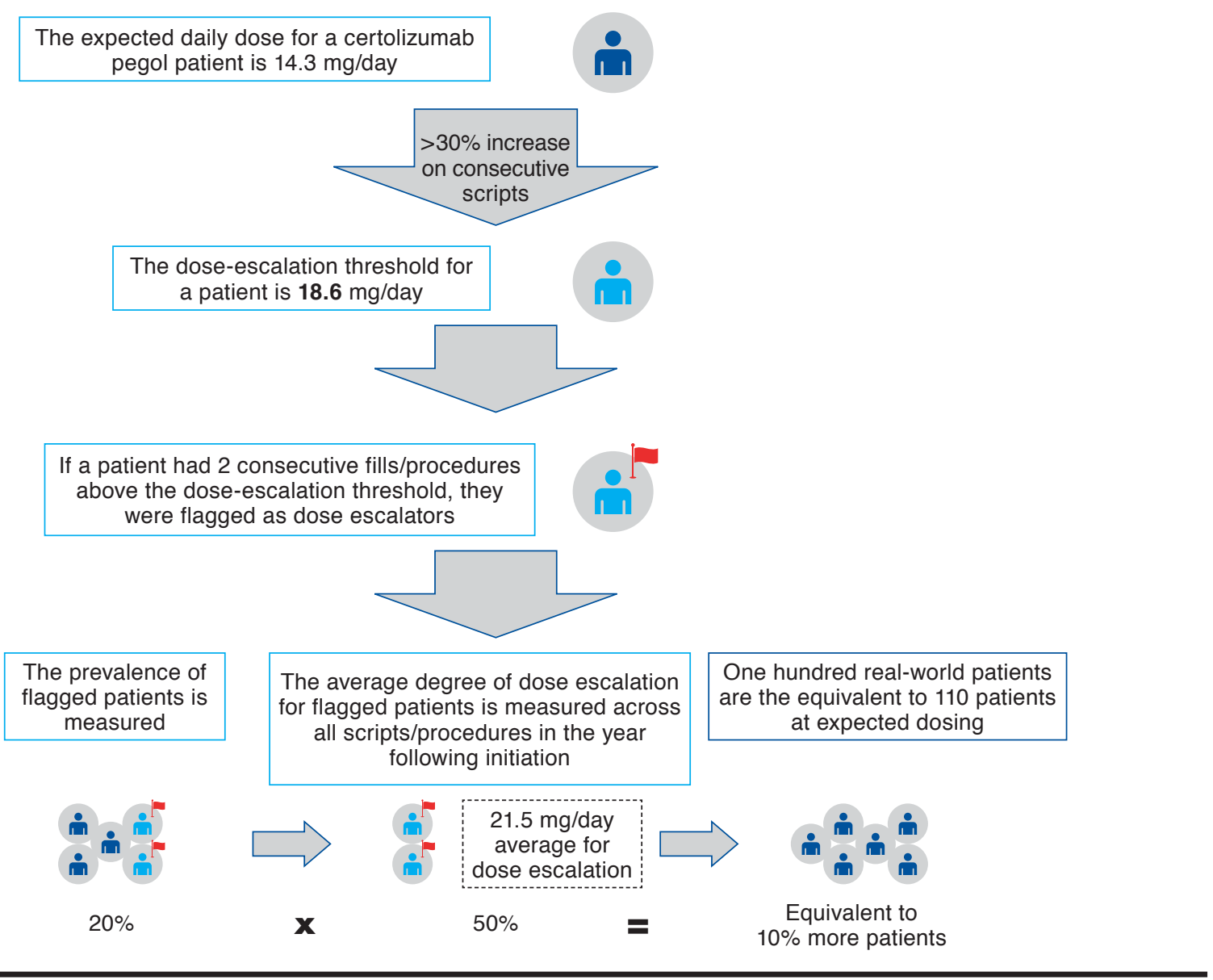

was estimated to be $20.9 \%$ per patient-year. Incidence rates of loss of response to vedolizumab maintenance therapy have been reported to be 47.9 per 100 person-years of follow-up in patients with CD and 39.8 per 100 person-years of follow-up in patients with UC. ${ }^{23}$

Recent studies have shown that the occurrence of suboptimal therapy among patients with IBD is common. ${ }^{24-26}$ Dose escalation in these cases has been shown to be efficacious, resulting in remission/response in patients who lost response to the initial biologic drug. ${ }^{23,27-36}$ However, these dose modifications may result in unexpected increased drug costs to payers in the United States.

There is a critical need to understand how physicians use TIMs to manage patients with IBD in clinical practice as dose escalation has the potential to affect the cost of treating these patients. Several new agents have been introduced to the marketplace to treat IBD including vedolizumab, ustekinumab, and tofacitinib. Limited data are available regarding comparative treatment patterns and rates of dose escalation of these newer agents in clinical practice. Therefore, the aim of this study was to evaluate the real-world prevalence and magnitude of dose escalation after induction dosing among TIMs approved for management of IBD and to determine the total number of patients that could have been treated with standard dosing given the prevalence of dose escalation in the treated population.

\section{Methods}

\section{Data Source}

This retrospective longitudinal study utilized Source Healthcare Analytics pharmacy and medical claims data between July 2015 and June 2018. This database contains more than 1 billion pharmacy transactions and more than 300 million physicians' services and hospitalizations that are representative of the insured population in the United States on a variety of demographic measures (e.g., age, gender, geographic distribution, and payment type). The medical claims data include inpatient and outpatient diagnosis information and procedure codes; pharmacy claims include patient prescription drug information. 


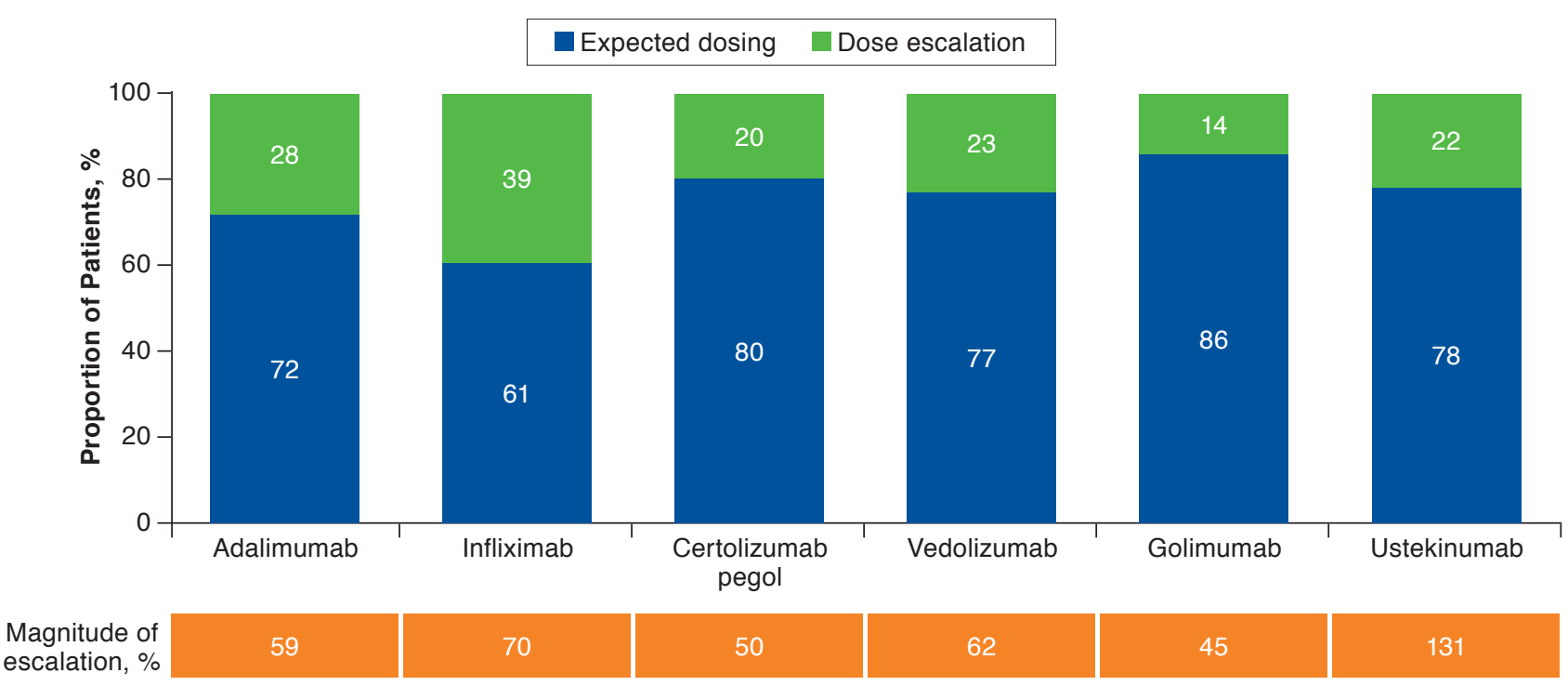

\section{Ethics}

This study was conducted in accordance with the ethical principles that have their origin in the current Declaration of Helsinki and was consistent with International Conference on Harmonization Good Clinical Practice and Good Epidemiology Practices and applicable regulatory requirements. The confidentiality of records that could identify individuals within the database was protected, respecting the privacy and confidentiality rules in accordance with applicable regulatory requirements.

\section{Study Population}

Commercially insured patients with evidence of initiation of one of the drugs of interest from July 1, 2015, to June 30, 2017, with claims for both medical and pharmacy services were selected for the analysis. Patients had to have at least 5 prescription fills/infusions during their respective first year of therapy (July 1, 2015, to June 2018). Drugs of interest included adalimumab, infliximab, infliximab biosimilars, certolizumab pegol (patient or physician-administered), ustekinumab (subcutaneous or infused), tofacitinib, golimumab (subcutaneous), and vedolizumab (infused). Patients taking infliximab biosimilars $(n=15)$ were removed from the analysis because of the low sample size. Tofacitinib was excluded because it did not receive U.S. Food and Drug Administration (FDA) approval for treatment of IBD until after the new patient start window; thus, no patients could be identified.

Patients were required to have at least 2 previous diagnoses (International Classification of Diseases, Ninth/Tenth Revision,
Clinical Modification [ICD-9/10-CM] codes; Appendix A, available in online article) for UC or CD before TIM initiation, with at least 1 occurring in the 12 months before the first prescription or infusion claim for a drug of interest. All included patients had evidence of claims at least 9 months before and 12 months after treatment initiation of a biologic of interest (i.e., biologic-naive or experienced). This increased the likelihood that patients were new initiators of the respective therapy and had previous activity in the dataset. Only patients with at least 5 prescriptions/infusions in the 12 months following their first fill/infusion were included in the analyses. This ensured a minimum level of persistence for each treatment and enabled identification of maintenance phase dosing used for a subset of the therapies. Prescription claims for patients were excluded from analysis after any evidence of nonpersistence. Nonpersistence was defined as a gap in therapy exceeding 1.5 times the standard dosing interval or days supply on the pharmacy claim. Patients with a previous claim for the initiating drug in the entire previous claim's history and those who switched between the branded and biosimilar version of infliximab during the study period were excluded.

\section{Dosing}

Expected Dosing. Expected daily dosing (EDD) or maintenance dosing for each therapy of interest is outlined in Appendix B (available in online article). EDD was derived from the prescribing information or initial maintenance treatment strength. ${ }^{37-42}$ EDD for infliximab was dictated by the initial 
treatment strength, for example, an infliximab patient treated with 3 vials initially had a baseline average daily dose (ADD) of $5.4 \mathrm{mg}$. Since administrative claims were used for the study of infused weight-based products (e.g., infliximab), the analysis accounted for whole vials used during treatment.

Average Daily Dosing. Average daily dosing (ADD) was calculated by dividing the quantity (in $\mathrm{mg}$ ) associated with the claim by the number of days between the fill/infusion date of the claim and that of the next claim. To minimize data entry errors, the ADD was calculated using the actual interval rather than the days supply listed on the claim. The last prescription for each patient was assigned the interval from the previous prescription (if needed).

Dose Escalation. Dose escalation was defined as an increase (minimum 30\%) in the ADD relative to the patient's expected maintenance dosage on an individual prescription/infusion (i.e., induction dosing was not included in the identification of dose escalation). ${ }^{43}$ Only postinduction claims for patients were examined for dose escalation. To avoid induction doses, patient claims were evaluated for dose escalation on the third prescription fill/infusion and beyond. Each claim was compared with the expected starting maintenance dose specific to its formulation for self-administered products, and intravenously infused products were compared with the initial patient-specific baseline maintenance dose. The criteria for dosage increase was a minimum increase in ADD of $30 \%$ on 2 consecutive prescriptions to avoid misclassifying patients (false positives) because of early refills or minor changes in the infusion schedule. Prescription fills/infusions that occurred within 4 days of another prescription fill/infusion were excluded from the analysis (presumed duplicates).

\section{Outcomes}

Patient Dose-Escalation Prevalence. Dose increases were evaluated in terms of the proportion of patients with dose escalation in the first 12 months after treatment initiation. Patient claims were followed during the first 12 months after treatment initiation until there was a gap in treatment. A gap in treatment was defined as 1.5 times the standard dosing interval for each product. Patient- and claim-level escalated dosing prevalence were determined using the escalation criteria. Patient prevalence was determined by dividing the patients that were flagged as dose escalators by the number of eligible patients. For claimlevel prevalence, claims were flagged as escalated if the patient met the escalation criteria and the claim surpassed the 30\% dose escalation threshold over the EDD. Prevalence was determined by dividing the dose escalation claim by all eligible claims. Only patient claims from the third prescription refill/infusion and beyond were evaluated in this analysis to avoid induction doses and to align with the dose escalation criteria.

\section{TABLE 1$)$ Summary of Patient-Level Dose Escalation}

\begin{tabular}{l|c|c|c|c}
\hline Drug & $\begin{array}{c}\text { Prevalence } \\
\text { of Dose } \\
\text { Escalation } \\
\text { (Patients, \%) }\end{array}$ & $\begin{array}{c}\text { Magnitude } \\
\text { of Dose } \\
\text { Escalation } \\
\text { (\%) }\end{array}$ & $\begin{array}{c}\text { Overall } \\
\text { Patient } \\
\text { Equivalence }\end{array}$ & $\begin{array}{c}\text { Patient } \\
\text { Sample }\end{array}$ \\
\hline Infliximab & 39 & 70 & 128 & 2,406 \\
\hline Adalimumab & 28 & 59 & 116 & 1,966 \\
\hline Vedolizumab & 23 & 62 & 114 & 1,745 \\
\hline Ustekinumab & 22 & 131 & 128 & 472 \\
\hline Certolizumab pegol & 20 & 50 & 110 & 285 \\
\hline Golimumab & 14 & 45 & 106 & 154 \\
\hline
\end{tabular}

Magnitude of Dose Escalation. The magnitude (average increase in dosing) that patients escalated was evaluated. Once patients were determined to be dose escalators, all claims following the induction period (after the third prescription refill) were assessed and compared with the EDD.

Overall Patient Equivalence. Calculation of patient equivalence associated with a therapeutic agent provides an estimate of the additional cost that payers may incur at the population level because it includes not only the prevalence of escalation but also the degree or magnitude of the escalated dose. Using the patient equivalence methodology, one can calculate how many additional patients could be treated over a period of time with the standard dose of therapy, and this estimate can assist with budget planning. Figure 1 illustrates the patient equivalence calculation for certolizumab pegol. The EDD for a patient treated with certolizumab pegol is $14.3 \mathrm{mg} / \mathrm{day}$. If there is more than a $30 \%$ increase in dose on 2 consecutive prescriptions, the dose escalation threshold is $18.6 \mathrm{mg} / \mathrm{day}$. If $20 \%$ of patients treated with certolizumab pegol have 2 consecutive prescriptions with more than a $30 \%$ increase in the expected dose, then these patients on average escalate their dose by $50 \%$ (to an average of $21.5 \mathrm{mg} /$ day). In this example, multiplying the prevalence of patients with dose escalation (0.2) by the magnitude of dose escalation in those patients (0.5) is equivalent to 0.1 or $10 \%$ more patients. Therefore, for each 100 patients treated over a year, the amount of drug that could treat 110 standardly dosed patients would be dispensed. In this study, the average degree of dose escalation was measured across all prescriptions of interest in the year following the induction period. Maintenance fills/infusions for dose escalation patients that were not above the escalation threshold were included in the average dose escalation calculation.

\section{Sensitivity Analysis}

Sensitivity testing was conducted on the 30\% dosing threshold and consecutive prescriptions to ensure the optimal dosage percentage increase (20\%-40\%) was being utilized. 


\section{FIGURE 3 Proportion of IBD Claims with Dose Escalation}

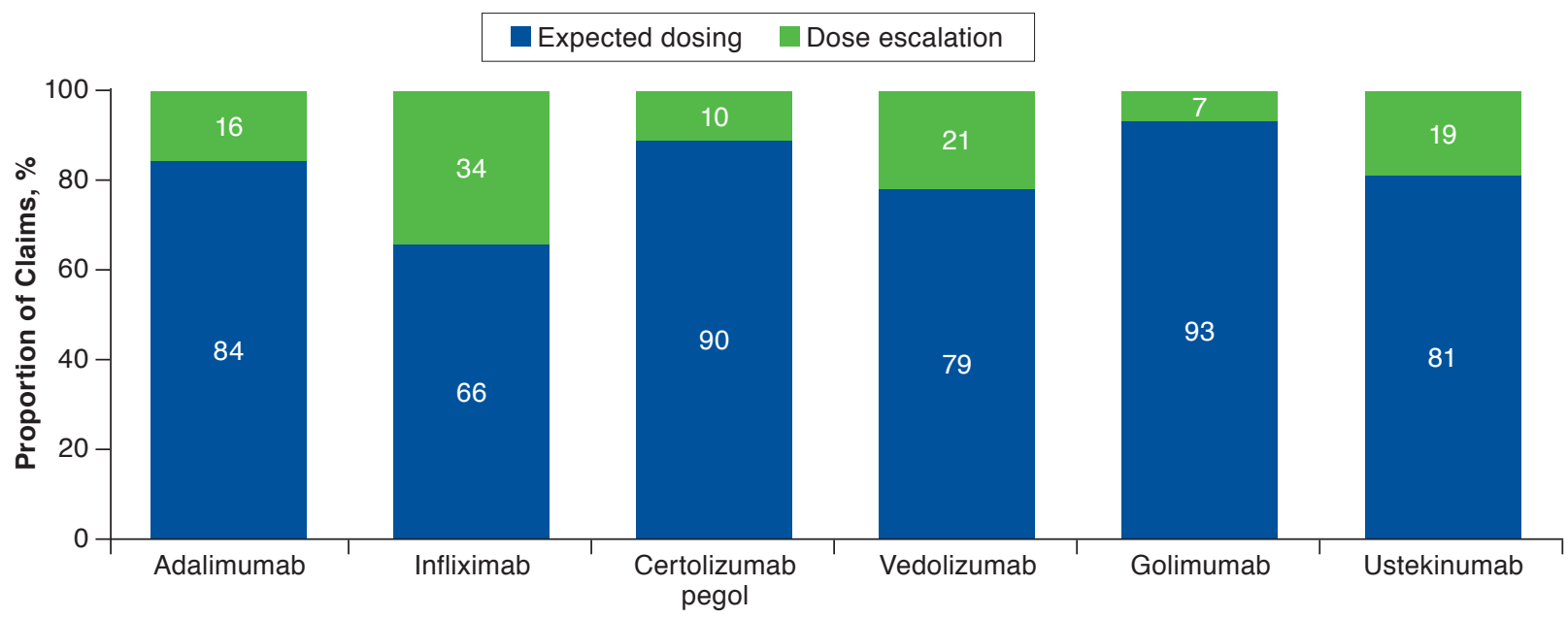

IBD = inflammatory bowel disease.

\section{Results}

\section{Patient Population}

The total number of patients included in the study was 7,028. Among them, 2,406, 1,966, 1,745, 472, 285, and 154 patients received infliximab, adalimumab, vedolizumab, ustekinumab, certolizumab pegol, and golimumab, respectively.

\section{Outcomes}

Prevalence of Dose Escalation. Drugs with short dosing intervals had the greatest difference in patient and claim dose-escalation prevalence. Adalimumab, certolizumab pegol, and golimumab all have 28-day dosing intervals, which are shorter than the other drugs of interest. Patient-level prevalence rates of dose escalation are shown in Figure 2 and Table 1. Infliximab-treated patients displayed the highest dose-escalation prevalence rate (39\%) followed by adalimumab (28\%), vedolizumab (23\%), ustekinumab (22\%), certolizumab pegol (20\%), and golimumab (14\%).

As shown in Figure 3, the claims-level prevalence rate of dose escalation was greatest for infliximab (34\%). The proportion of vedolizumab, ustekinumab, adalimumab, certolizumab pegol, and golimumab claims meeting dose escalation criteria was $21 \%, 19 \%, 16 \%, 10 \%$, and $7 \%$, respectively.

Magnitude of Dose Escalation. The magnitude of dose escalation was greatest in ustekinumab-treated patients (131\%) followed by infliximab (70\%), vedolizumab (62\%), adalimumab (59\%), certolizumab pegol (50\%), and golimumab (45\%; Figure 2 and Table 1).

Patient Equivalence. Patient equivalence data are shown in Figure 4. Infliximab and ustekinumab display the highest patient equivalence (128 each); adalimumab and vedolizumab exhibit similar patient equivalents (116 and 114, respectively); and certolizumab pegol and golimumab display the lowest patient equivalence (110 and 106, respectively). The realworld dosing of 100 patients is equivalent to treating 106, $110,114,116,128$, and 128 patients with standard dosing for golimumab, certolizumab pegol, vedolizumab, adalimumab, ustekinumab, and infliximab, respectively.

\section{Sensitivity Analysis}

Sensitivity testing was conducted on the 30\% dosing threshold to ensure the optimal dosage percentage increase (20\%-40\%) was being utilized. For all drugs of interest, the magnitude of dose escalation increased with the increased dosing threshold in the sensitivity analysis, while the prevalence rates decreased. The order of TIMs in terms of which drugs had the highest versus lowest rates remained the same at the 30\% and $40 \%$ thresholds for prevalence and 20\% and 30\% thresholds for magnitude.

\section{Discussion}

This study provides valuable insights on the real-world prevalence of dose escalation of TIMs in the treatment of IBD. To our knowledge this is the first study to use the calculation of patient equivalency to quantify dose escalation. In this study, infliximab-treated patients displayed the highest dose-escalation prevalence rate (39\%). Adalimumab-treated patients were less likely to dose escalate than infliximab patients ( $28 \%$ vs. $39 \%$, respectively). All other treated gastroenterology patients were less likely to dose escalate on their respective treatment drugs $(\leq 23 \%)$. Observed rates of dose escalation in this study 


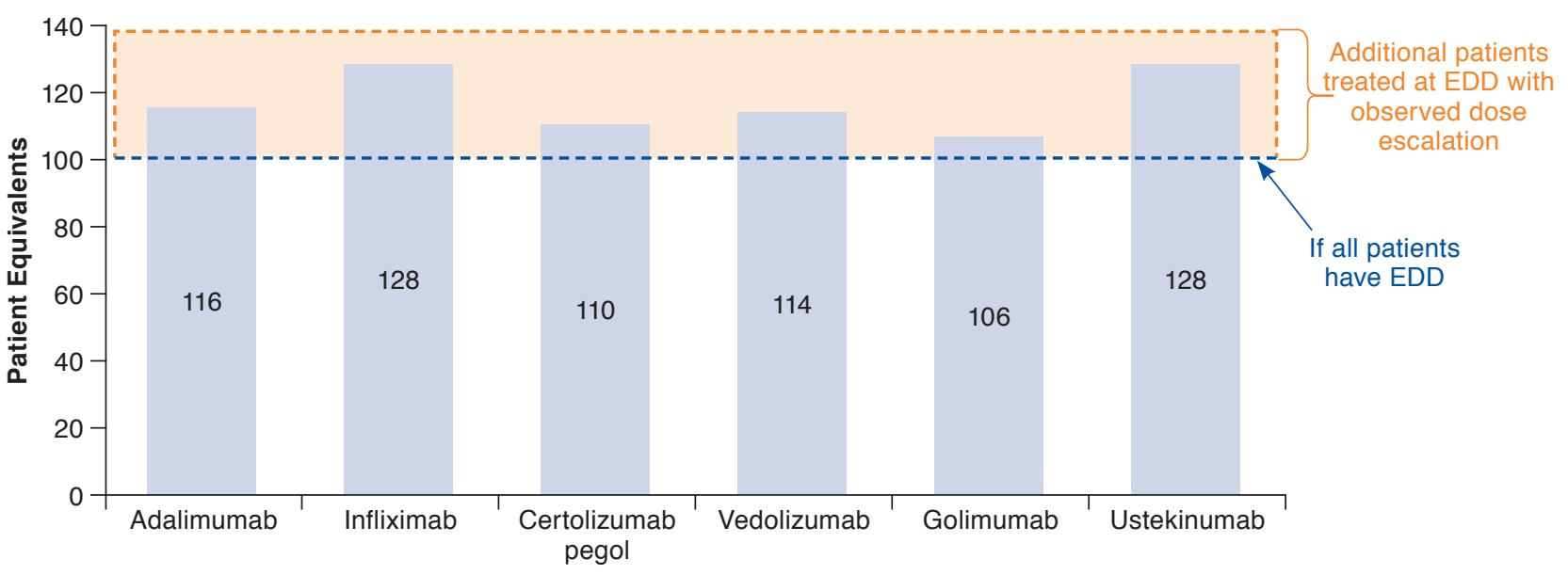

$E D D=$ expected daily dosing

are within the range of those reported in previous real-world observational studies. $24,27,36,44-46$

This study adds insight on the magnitude of dose escalation and the patient equivalency to standard dosing based on the prevalence and magnitude of dose escalation for each drug, providing a method to predict drug utilization. This novel approach can be used by a health care system or payer to evaluate the true cost of therapy in combination with discounts or rebates. For example, if patient equivalence at observed dosing of a therapy is 116 (which is greater than 100), then the cost of this therapy would represent a 16\% increase in the budgeted cost, assuming that the budget was based on standard dosing. This would mean that the cost of treating 100 patients would be equivalent to the cost of treating 116 patients. Thus, comparing the equivalency of treating patients using observed prevalence and magnitude of dose escalations to treating 100 patients with standard dosing highlights the potential increases in costs and is important information for payers.

The observed rates of dose escalations in the present study highlight the need and importance of dose optimization and individualizing therapy in the clinical management of patients with IBD. Dose escalation has been shown to be an effective option in patients with IBD who have secondary loss of response to their biologic drug treatment. ${ }^{23,30,31,33,34}$

A few recent studies have suggested that therapeutic drug monitoring and testing of anti-TNF antibody levels in patients with IBD may lead to better clinical outcomes and help guide the timing and appropriateness of dose escalation and deescalation of therapy. ${ }^{47-49}$ From a clinical management perspective, testing for anti-TNF antibody and drug concentrations is deemed appropriate at the end of induction therapy for primary nonresponders, in secondary nonresponders, and at least once during the first year of maintenance therapy. ${ }^{48}$ Based on results, physicians could then increase the anti-TNF dose if drug concentrations were too low to be effective or switch to a different type of anti-TNF agent if antidrug antibodies were high. If these modifications to therapy fail, then changing to a biologic with a different mechanism of action would be warranted. ${ }^{48}$ This management strategy may limit unnecessary dose escalation while providing appropriate treatment to achieve desired clinical outcomes.

One strength of the current study is that it includes both medical and pharmacy prescriptions so that a robust analysis of the frequency of dose escalation may be conducted in the United States. Another strength is the use of a patient-equivalent approach to assess drug utilization; use of this approach provides a means to determine the amount of dose escalation in a consistent manner across therapies.

\section{Limitations}

Several limitations of the analysis need to be considered when interpreting the results. This study may overestimate actual dose escalation as there are many reasons why a claim may be submitted multiple times regardless of the actual use of the medication. However, to minimize overestimation of dose escalation due to patients filling their prescription early, we used 2 consecutive claims in the analysis. Other behavioral (e.g., patient travel or change in residence) or institutional scenarios (e.g., multiple submissions of the same claim) would likely be similar across products and, therefore, relative results would remain the same. The presence of a claim for a filled prescription does not indicate that the patient used the drug or that it 
was taken on or near the date of fill; however, it is expected that this potential issue would be presented equally among the drugs of interest. Drug quantity used in the dosing evaluation was based on quantities billed to the patient's health plan, not necessarily the dose used for treatment. It is not possible to verify the dose used for treatment because the instructions that physicians provide to their patients are not available on claims data. Determination of drug quantity is particularly sensitive for a product like infliximab where the unit of measure is vials (as opposed to syringes or pens for adalimumab). The evaluation examined claims data used for billing health plans and may be subject to data errors (e.g., miscoding); however, these errors are likely to have a similar prevalence among the drugs of interest. Finally, it should be noted that this study was not able to analyze dose escalation among patients taking tofacitinib because tofacitinib did not receive approval as a treatment for IBD by the FDA until after the start of the new patient study period, so there were no patients captured in the database at the time of this analysis. Furthermore, vedolizumab was approved for the treatment of CD and UC in 2014, ustekinumab was approved for CD in 2016 and for UC in 2019, and the recent approval of these medications may have affected the proportion of patients represented in this study. Since treatment patterns may change with increased use of these newer agents, future studies are needed to examine results with tofacitinib, vedolizumab, and ustekinumab as well as infliximab biosimilars to provide a more comprehensive analysis of dose escalation.

\section{Conclusions}

Dose escalation in patients with IBD occurred with all TIMs evaluated in this study with varying degrees of prevalence and magnitude. On average, patients with IBD who were treated with infliximab and ustekinumab consumed more medication than patients on other therapies in the study due to the prevalence and magnitude of dose escalation. Patients who escalate dosing may represent an unexpected cost to payers, and realworld utilization patterns of TIMs suggest that dose escalation should be considered when assessing the overall cost of care for a patient.

\section{Authors}

RAHEL EHRENBERG, BS, and BRYAN MCDONALD, BS, IQVIA, Cambridge, Massachusetts. JENNY GRIFFITH, PharmD, and CINDY THEIGS, RPh, AbbVie, North Chicago, Illinois.

AUTHOR CORRESPONDENCE: Jenny Griffith, PharmD, Health Economics and Outcomes Research, AbbVie, 8457 Stone Ledge Dr., Edwardsville, IL 62025. Tel.: 618.692.6419;

E-mail: jenny.griffith@abbvie.com.

\section{DISCLOSURES}

Financial support for this study was provided by AbbVie, which participated in study design, research, data collection, analysis and interpretation of data writing, reviewing, and approving the publication. All authors contributed to the development of the publication and maintained control over the final content. Ehrenberg and McDonald are employees of IQVIA, which received funding from AbbVie to participate in this research. Griffith and Theigs are employed by AbbVie and may own stock or stock options in AbbVie.

\section{ACKNOWLEDGMENTS}

Medical writing support was provided by Joann Hettasch of JK Associates, a member of the Fishawack Group of Companies, Conshohocken, PA. This support was funded by AbbVie.

\section{REFERENCES}

1. Chaparro M, Gisbert JP. Maintenance therapy options for ulcerative colitis. Expert Opin Pharmacother. 2016;17(10):1339-49.

2. Lichtenstein GR, Loftus EV, Isaacs KL, Regueiro MD, Gerson LB, Sands BE. ACG clinical guideline: management of Crohn's disease in adults. Am J Gastroenterol. 2018;113(4):481-517.

3. Maaser C, Sturm A, Vavricka SR, et al. ECCO-ESGAR guideline for diagnostic assessment in IBD part 1: initial diagnosis, monitoring of known IBD, detection of complications. J Crohns Colitis. 2019;13(2):144-64

4. Fumery M, Pineton de Chambrun G, Stefanescu C, et al. Detection of dysplasia or cancer in $3.5 \%$ of patients with inflammatory bowel disease and colonic strictures. Clin Gastroenterol Hepatol. 2015;13(10):1770-75.

5. Ordas I, Eckmann L, Talamini M, Baumgart DC, Sandborn WJ. Ulcerative colitis. Lancet. 2012;380(9853):1606-19.

6. Torres J, Billioud V, Sachar DB, Peyrin-Biroulet L, Colombel JF. Ulcerative colitis as a progressive disease: the forgotten evidence. Inflamm Bowel Dis. 2012;18(7):1356-63.

7. Peyrin-Biroulet L, Loftus EV, Jr, Colombel JF, Sandborn WJ. The natural history of adult Crohn's disease in population-based cohorts. Am J Gastroenterol. 2010;105(2):289-97.

8. Cohen-Mekelburg S, Schneider Y, Gold S, et al. Risk of early colorectal cancers needs to be considered in inflammatory bowel disease care. Dig Dis Sci. 2019;64(8):2273-79.

9. Choi CR, Al Bakir I, Ding NJ, et al. Cumulative burden of inflammation predicts colorectal neoplasia risk in ulcerative colitis: a large single-centre study. Gut. 2019;68(3):414-22.

10. Gibson PR, Vaizey C, Black CM, et al. Relationship between disease severity and quality of life and assessment of health care utilization and cost for ulcerative colitis in Australia: a cross-sectional, observational study. J Crohns Colitis. 2014;8(7):598-606.

11. Cohen BL, Zoega H, Shah SA, et al. Fatigue is highly associated with poor health-related quality of life, disability and depression in newly-diagnosed patients with inflammatory bowel disease, independent of disease activity. Aliment Pharmacol Ther. 2014;39(8):811-22.

12. Romberg-Camps MJ, Bol Y, Dagnelie PC, et al. Fatigue and health-related quality of life in inflammatory bowel disease: results from a populationbased study in the Netherlands: the IBD-South Limburg cohort. Inflamm Bowel Dis. 2010;16(12):2137-47.

13. Louis E, Lofberg R, Reinisch W, et al. Adalimumab improves patientreported outcomes and reduces indirect costs in patients with moderate to severe Crohn's disease: results from the CARE trial. J Crohns Colitis. 2013;7(1):34-43

14. Mikocka-Walus A, Knowles SR, Keefer L, Graff L. Controversies revisited: a systematic review of the comorbidity of depression and anxiety with inflammatory bowel diseases. Inflamm Bowel Dis. 2016;22(3):752-62. 
15. Travis S, Feagan BG, Peyrin-Biroulet L, et al. Effect of adalimumab on clinical outcomes and health-related quality of life among patients with ulcerative colitis in a clinical practice setting: results from InspirADA. J Crohns Colitis. 2017;11(11):1317-25.

16. Van Assche G, Peyrin-Biroulet L, Sturm A, et al. Burden of disease and patient-reported outcomes in patients with moderate to severe ulcerative colitis in the last 12 months - multicenter European cohort study. Dig Liver Dis. 2016;48(6):592-600

17. Casellas F, Robles V, Borruel N, et al. Restoration of quality of life of patients with inflammatory bowel disease after one year with antiTNFa treatment. J Crohns Colitis. 2012;6(9):881-86.

18. Yarlas A, Rubin DT, Panes J, et al. Burden of ulcerative colitis on functioning and well-being: a systematic literature review of the SF-36(R) health survey. J Crohns Colitis. 2018;12(5):600-09.

19. Panaccione R, Ghosh S. Optimal use of biologics in the management of Crohn's disease. Therap Adv Gastroenterol. 2010;3(3):179-89.

20. Park SC, Jeen YT. Current and emerging biologics for ulcerative colitis. Gut Liver. 2015;9(1):18-27.

21. Roda G, Jharap B, Neeraj N, Colombel JF. Loss of response to antiTNFs: definition, epidemiology, and management. Clin Transl Gastroenterol. 2016;7(1):e135

22. Qiu Y, Chen BL, Mao R, et al. Systematic review with meta-analysis: loss of response and requirement of anti-TNFa dose intensification in Crohn's disease. J Gastroenterol. 2017;52(5):535-54.

23. Peyrin-Biroulet L, Danese S, Argollo M, et al. Loss of response to vedolizumab and ability of dose intensification to restore response in patients with Crohn's disease or ulcerative colitis: a systematic review and meta-analysis. Clin Gastroenterol Hepatol. 2019;17(5):838-46.e2.

24. Khan S, Rupniewska E, Neighbors M, Singer D, Chiarappa J, Obando C. Real-world evidence on adherence, persistence, switching and dose escalation with biologics in adult inflammatory bowel disease in the United States: a systematic review. J Clin Pharm Ther. 2019;44(4):495-507.

25. Lindsay JO, Armuzzi A, Gisbert JP, et al. Indicators of suboptimal tumor necrosis factor antagonist therapy in inflammatory bowel disease. Dig Liver Dis. 2017:49(10):1086-91.

26. Patel H, Lissoos T, Rubin DT. Indicators of suboptimal biologic therapy over time in patients with ulcerative colitis and Crohn's disease in the United States. PLoS One. 2017;12(4):e0175099.

27. Baert F, Glorieus E, Reenaers C, et al. Adalimumab dose escalation and dose de-escalation success rate and predictors in a large national cohort of Crohn's patients. J Crohns Colitis. 2013;7(2):154-60.

28. Black CM, Yu E, McCann E, Kachroo S. Dose escalation and healthcare resource use among ulcerative colitis patients treated with adalimumab in English hospitals: an analysis of real-world data. PLoS One. 2016;11(2):e0149692

29. Chan W, Lynch N, Bampton P, et al. Entyvio lengthen dose-interval study: lengthening vedolizumab dose interval and the risk of clinical relapse in inflammatory bowel disease. Eur J Gastroenterol Hepatol. 2018;30(7):735-40.

30. Dubinsky MC, Rosh J, Faubion WA, Jr., et al. Efficacy and safety of escalation of adalimumab therapy to weekly dosing in pediatric patients with Crohn's disease. Inflamm Bowel Dis. 2016;22(4):886-93.

31. Duveau N, Nachury M, Gerard R, et al. Adalimumab dose escalation is effective and well tolerated in Crohn's disease patients with secondary loss of response to adalimumab. Dig Liver Dis. 2017;49(2):163-69.

32. Engel T, Ungar B, Yung DE, Ben-Horin S, Eliakim R, Kopylov U.

Vedolizumab in IBD-lessons from real-world experience; a systematic review and pooled analysis. J Crohns Colitis. 2018;12(2):245-57.
33. Ma C, Huang V, Fedorak DK, et al. Adalimumab dose escalation is effective for managing secondary loss of response in Crohn's disease. Aliment Pharmacol Ther. 2014:40(9):1044-55.

34. Taxonera C, Barreiro-de Acosta M, Calvo M, et al. Infliximab dose escalation as an effective strategy for managing secondary loss of response in ulcerative colitis. Dig Dis Sci. 2015;60(10):3075-84.

35. Taxonera C, Olivares D, Mendoza JL, Diaz-Rubio M, Rey E. Need for infliximab dose intensification in Crohn's disease and ulcerative colitis. World J Gastroenterol. 2014;20(27):9170-77.

36. Tkacz J, Lofland JH, Vanderpoel J, Ruetsch C. Infliximab dosing patterns in a sample of patients with Crohn's disease: results from a medical chart review. Am Health Drug Benefits. 2014;7(2):87-93.

37. Humira (adalimumab) injection, for subcutaneous use. AbbVie. January 2019. Available at: https://www.rxabbvie.com/pdf/humira.pdf. Accessed March 2, 2020.

38. Simponi (golimumab) injection, for subcutaneous use. Janssen Biotech October 2019. Available at: http://www.janssenlabels.com/package-insert/ product-monograph/prescribing-information/SIMPONI-pi.pdf. Accessed March 2, 2020.

39. Remicade (infliximab) lyophilized concentrate for injection, for intravenous use. Janssen Biotech. October 2017. Available at: http://www.janssenlabels.com/package-insert/product-monograph/prescribing-information/ REMICADE-pi.pdf. Accessed March 2, 2020.

40. Entyvio (vedolizumab) for injection, for intravenous use. Takeda Pharmaceuticals. May 2019. Available at: https://general.takedapharm.com/ ENTYVIOPI. Accessed March 2, 2020.

41. Stelara (ustekinumab) injection, for subcutaneous or intravenous use. Janssen Biotech. January 2020. Available at: http://www.janssenlabels.com/ package-insert/product-monograph/prescribing-information/STELARA-pi. pdf. Accessed March 2, 2020.

42. Cimzia (certolizumab pegol) for injection, for subcutaneous use. UCB. September 2019. Available at: https://www.cimzia.com/themes/custom/cimzia/ docs/CIMZIA_full_prescribing_information.pdf. Accessed March 2, 2020.

43. Ollendorf DA, Klingman D, Hazard E, Ray S. Differences in annual medication costs and rates of dosage increase between tumor necrosis factorantagonist therapies for rheumatoid arthritis in a managed care population. Clin Ther. 2009;31(4):825-35.

44. Einarson TR, Bereza BG, Ying Lee X, Lelli F. Dose escalation of biologics in Crohn's disease: critical review of observational studies. Curr Med Res Opin. 2017;33(8):1433-49

45. Gisbert JP, Panes J. Loss of response and requirement of infliximab dose intensification in Crohn's disease: a review. Am J Gastroenterol. 2009;104(3):760-67.

46. O'Donnell S, Stempak JM, Steinhart AH, Silverberg MS. Higher rates of dose optimisation for infliximab responders in ulcerative colitis than in Crohn's disease. J Crohns Colitis. 2015;9(10):830-36.

47. Kelly OB, Donnell SO, Stempak JM, Steinhart AH, Silverberg MS Therapeutic drug monitoring to guide infliximab dose adjustment is associated with better endoscopic outcomes than clinical decision making alone in active inflammatory bowel disease. Inflamm Bowel Dis. 2017;23(7):1202-09.

48. Melmed GY, Irving PM, Jones J, et al. Appropriateness of testing for antitumor necrosis factor agent and antibody concentrations, and interpretation of results. Clin Gastroenterol Hepatol. 2016;14(9):1302-09.

49. Velayos FS, Kahn JG, Sandborn WJ, Feagan BG. A test-based strategy is more cost effective than empiric dose escalation for patients with Crohn's disease who lose responsiveness to infliximab. Clin Gastroenterol Hepatol. 2013;11(6):654-66. 


\begin{tabular}{l|l|l}
\hline \multicolumn{1}{c|}{ APPENDIX A } & $\begin{array}{l}\text { Diagnostic Codes for Ulcerative } \\
\text { Colitis and Crohn Disease }\end{array}$ \\
\hline Indication & ICD-9-CM & \multicolumn{1}{c}{ ICD-10-CM } \\
\hline Ulcerative colitis & $556 . \mathrm{xx}$ & $\mathrm{K} 51.0, \mathrm{~K} 51.2, \mathrm{~K} 51.3, \mathrm{~K} 51.4$, \\
& & $\mathrm{K} 51.5, \mathrm{~K} 51.8, \mathrm{~K} 51.9, \mathrm{M} 06.2$, \\
& & $\mathrm{M} 06.3, \mathrm{M} 07.5, \mathrm{M} 09.2, \mathrm{M} 46.5$, \\
& & $\mathrm{K} 48.8$ \\
\hline Crohn disease & $555 . \mathrm{xx}$ & $\mathrm{M} 07.4, \mathrm{~K} 50.1, \mathrm{~K} 509.1$ \\
\hline
\end{tabular}

\begin{tabular}{|c|c|c|c|c|}
\hline Therapy & Interval (Days) & Dose & EDD (mg) & $\begin{array}{l}\text { Frequency of Administration } \\
\text { for Weight-Based TIMs }\end{array}$ \\
\hline Adalimumab & 28 & $80 \mathrm{mg}$ & 2.9 & NA \\
\hline Certolizumab pegol & 28 & $400 \mathrm{mg}$ & 14.3 & NA \\
\hline Vedolizumab & 56 & $300 \mathrm{mg}$ & 5.4 & NA \\
\hline Golimumab & 28 & $100 \mathrm{mg}$ & 3.6 & NA \\
\hline Ustekinumab & 56 & $90 \mathrm{mg}$ & 1.6 & NA \\
\hline Infliximab & 56 & $5 \mathrm{mg} / \mathrm{kg}$ & Varies by weight & Every 8 weeks \\
\hline
\end{tabular}

